\title{
A case of $X$-linked hypophosphatemic rickets: complications and the therapeutic use of cinacalcet
}

\author{
Helge Ræder ${ }^{1,2}$, Nick Shaw ${ }^{3}$, Coen Netelenbos ${ }^{4}$ and Robert Bjerknes ${ }^{1,2}$ \\ ${ }^{1}$ Section for Pediatrics, Department of Clinical Medicine, University of Bergen, 5021 Bergen, Norway, ${ }^{2}$ Department of Pediatrics, Haukeland University \\ Hospital, 5021 Bergen, Norway, ${ }^{3}$ Department of Endocrinology, Birmingham Children's Hospital, Birmingham, UK and ${ }^{4}$ Department of Endocrinology, \\ Academic Hospital Vrije Universiteit Amsterdam, Amsterdam, The Netherlands \\ (Correspondence should be addressed to H Rader; Email: helge.rader@uib.no)
}

\begin{abstract}
In hypophosphatemic rickets, there are both inherited and acquired forms, where X-linked dominant hypophosphatemic rickets (XLH) is the most prevalent genetic form and caused by mutations in the phosphate-regulating endopeptidase (PHEX) gene. XLH is associated with growth retardation and bone deformities. The renal tubular cells have an important role in calcium and phosphate metabolism, where the $1 \alpha$-hydroxylase enzyme metabolizes the conversion of $25(\mathrm{OH})$-vitamin D to potent $1,25(\mathrm{OH})_{2^{-}}$ vitamin D, whereas the sodium-phosphate transporter controls tubular phosphate reabsorption. The pathophysiological defect in XLH is speculated to cause an increase in a circulating phosphate regulating hormone termed phosphatonin (fibroblast growth factor 23 is the primary phosphatonin candidate), which leads to inhibition of $1 \alpha$-hydroxylase, and simultaneously to inhibition of the sodium-phosphate transporter domain NPT2c leading to parathyroid hormone-independent phosphaturia. Hence, current treatment of XLH is 1,25 $(\mathrm{OH})_{2}$-vitamin D or the vitamin D analog alfacalcidol and elementary phosphorus. Unfortunately, patients with XLH may develop nephrocalcinosis, secondary or tertiary hyperparathyroidism, and in some situations also hypertension and cardiovascular abnormalities. We describe a patient with XLH caused by a novel missense mutation in the PHEX gene, who on treatment with alfacalcidol and oral phosphate had normal growth and minimal bone deformities, but who subsequently developed moderate nephrocalcinosis, significant hyperparathyroidism, hypercalcemia, renal failure, and hypertension. We also report the use of the calcimimetic drug cinacalcet in the successful treatment of hypercalcemia and hyperparathyroidism.
\end{abstract}

European Journal of Endocrinology 159 S101-S105

\section{Introduction}

Rickets and osteomalacia are disorders that result from decreased mineralization of bone matrix or osteoid caused by deficiencies of calcium or phosphorus or both. Rickets is usually divided into hypocalcemic and hypophosphatemic rickets, based on the deficient mineral. In hypophosphatemic rickets, there are both inherited and acquired forms, where X-linked dominant hypophosphatemic rickets (XLH) is the most prevalent genetic form and caused by mutations in the PHEX gene. Such mutations are speculated to increase the levels of phosphatonins by reduced degradation or increased production (1). Phosphatonins are circulating factors, mainly produced in bone, that act on proximal renal tubular cells to increase phosphate wasting by down-regulation of sodium-phosphate co-transporters (1). In addition they reduce the activity of the

This paper was presented at the 5th Ferring International Paediatric Endocrinology Symposium, Baveno, Italy (2008). Ferring Pharmaceuticals has supported the publication of these proceedings.
$1 \alpha$-hydroxylase enzyme leading to inappropriately normal levels of $1,25(\mathrm{OH})_{2}$ vitamin D3 in the face of hypophosphatemia (1). Fibroblast growth factor 23 (FGF23) is considered to be the major phosphatonin, and increased levels have been observed in XLH (2).

Untreated XLH is associated with growth retardation and bone deformities, whereas treatment with oral phosphate and vitamin D preparation may improve growth (3) but is associated with complications including nephrocalcinosis (3), hyperparathyroidism (4), hypertension (5), and cardiovascular abnormalities (6). Hyperparathyroidism may be an early event in the development of other complications, in particular hypertension (5), and measures to prevent hyperparathyroidism include the appropriate dosing of phosphate and vitamin D preparation (4). Recently, the calcimimetic drug cinacalcet has been reported to be effective in the treatment of secondary hyperparathyroidism in patients receiving hemodialysis (7), and there is some preliminary evidence of a beneficial effect in XLH (8).

We describe a patient with XLH, caused by a novel missense mutation in the PHEX gene, who on treatment 

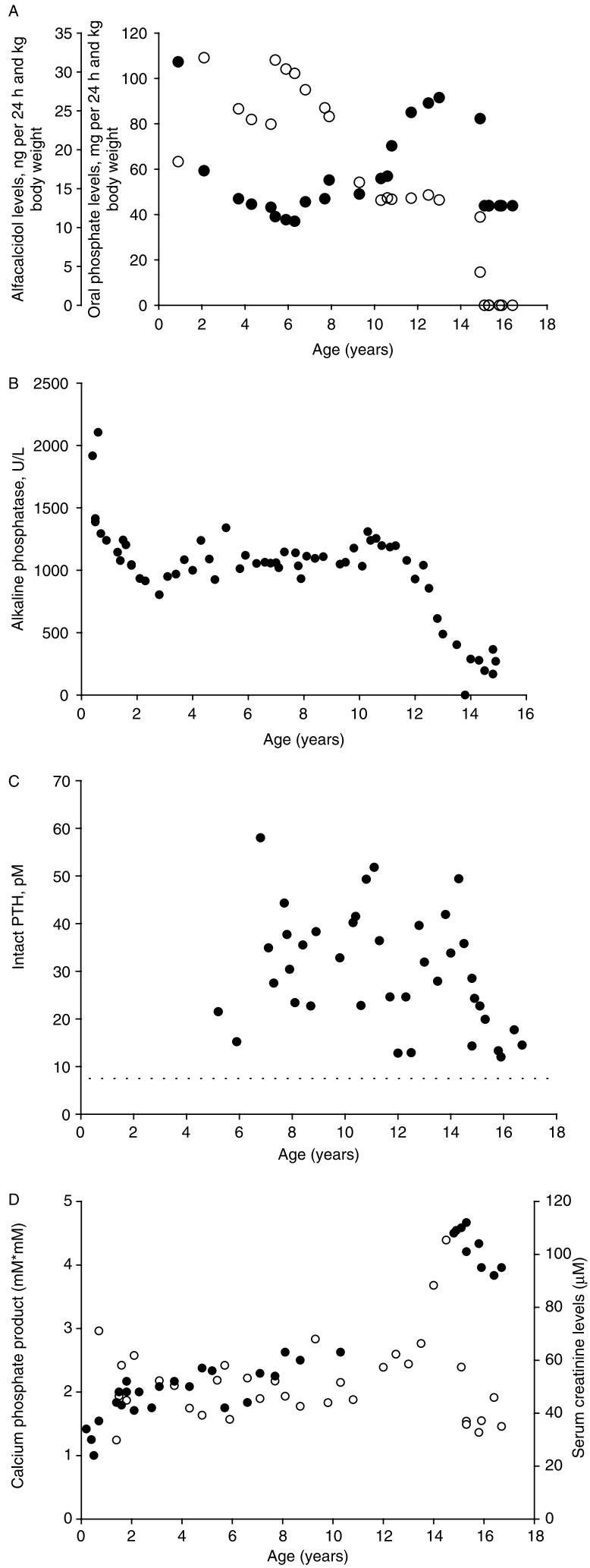

with alfacalcidol and oral phosphate had normal growth and minimal bone deformities, but who subsequently developed moderate nephrocalcinosis, significant hyperparathyroidism, hypercalcemia, renal failure, and hypertension. We also report the use of the calcimimetic drug cinacalcet in treatment of hypercalcemia and hyperparathyroidism in this patient.

\section{Methods}

The studies were performed according to the Declaration of Helsinki of 1975, as revised in 2000. Informed consent was obtained from all subjects or their parents.

All coding exon and exon-intron boundaries of the PHEX gene were screened for mutations (Amplexa Genetics, Odense, Denmark).

\section{Case history}

The patient was a healthy girl by birth, born at term, birth weight $2960 \mathrm{~g}$ (just below 10th centile), who, by 5 months of age, developed subnormal serum phosphate levels and the characteristic findings of rickets. Due to the family history, the diagnosis of XLH was suspected, and she was started on treatment with oral phosphate doses six times daily and alfacalcidol (Fig. 1A), and her levels of alkaline phosphatase improved (Fig. 1B). At the age of five, she had an intermittent clinical and radiological progression of rickets. Her parathyroid hormone (PTH) levels remained elevated, whereas her calcium phosphate product and serum creatinine levels were normal in her first decade (Fig. 1C and D). She had a moderate and stable nephrocalcinosis as evaluated by ultrasonographic examinations of her kidneys (grade II-III) (9).

Her mother had been diagnosed with hypophosphatemic rickets and was of short stature and had developed genu varum (Fig. 2A). The parents of the mother had reportedly normal serum phosphate levels. A 4-year old younger brother of the patient was considered to be healthy (he had by 1 year of age a phosphate level of $1.46 \mathrm{mM}$, a calcium level of $2.48 \mathrm{mM}$ and alkaline phosphatase level of $415 \mathrm{U} / \mathrm{l})$. No other relatives were clinically affected (Fig. 1B). We found a novel missense mutation, c.914T $>C$ (L305P), in exon 8 of the PHEX gene, co-segregating with disease (Fig. 2B). This amino acid substitution was phylogenetically conserved and occurred in the extracellular domain of the PHEX protein, and no known polymorphisms have been reported in this position to date to our knowledge.

Figure 1 Treatment dosages and laboratory results. (A) Dosages of alfacalcidol (filled circles) and oral phosphate (open circles). (B) Alkaline phosphatase levels. The upper normal limit is $1000 \mathrm{U} / \mathrm{l}$. (C) Intact PTH levels. The normal reference interval is $0.7-7.5 \mathrm{pM}$. (D) Calcium phosphate product (open circles) and serum creatinine levels (filled circles). 

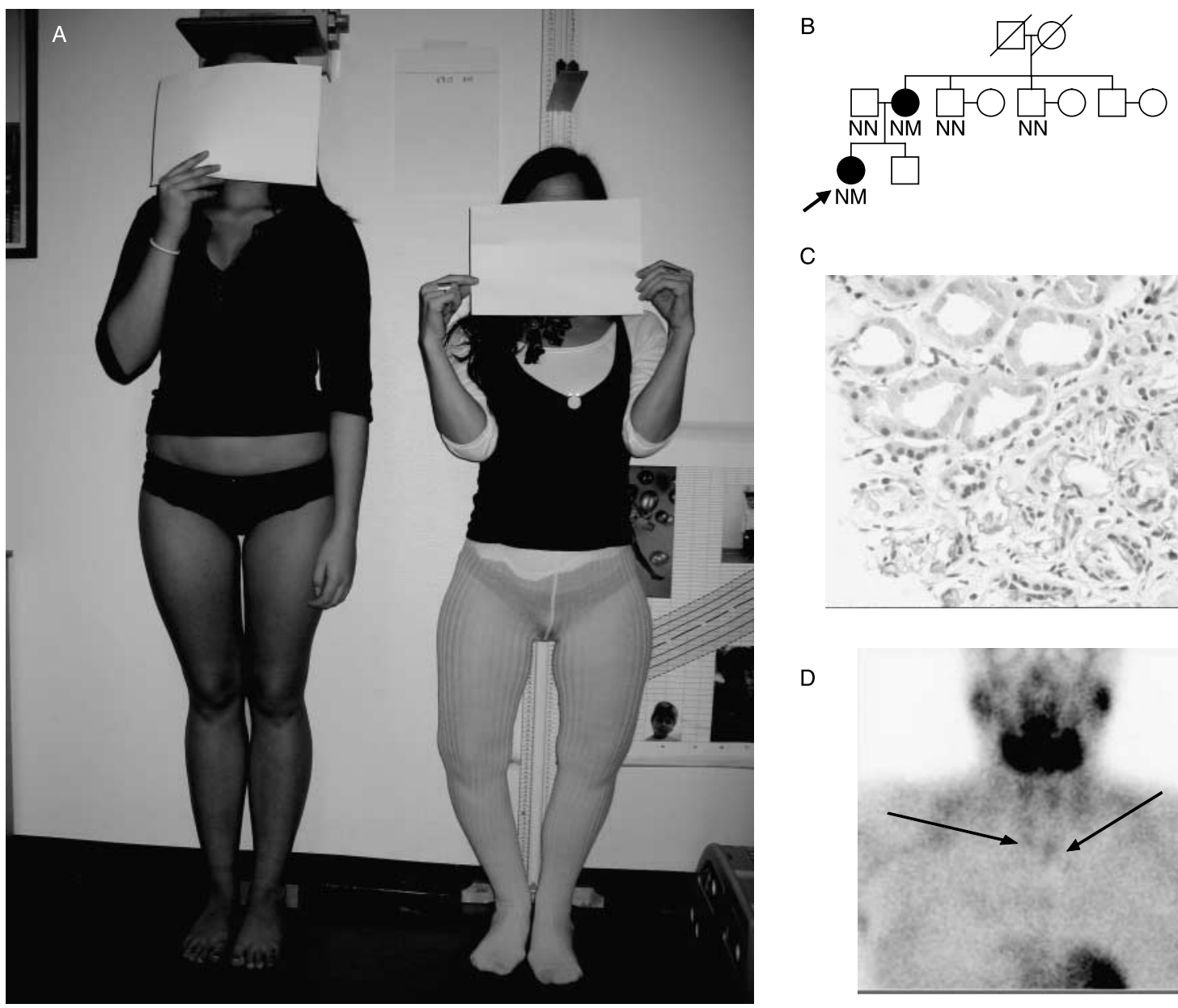

C

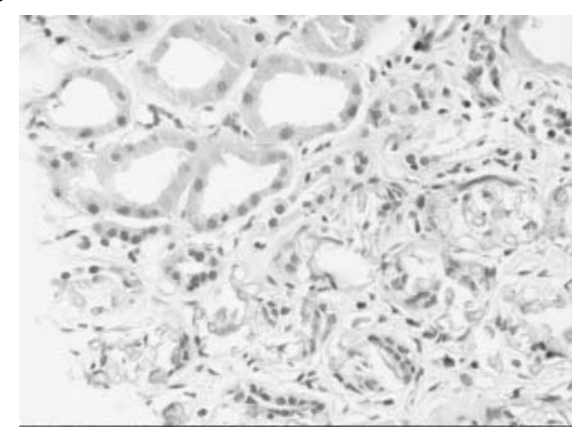

D

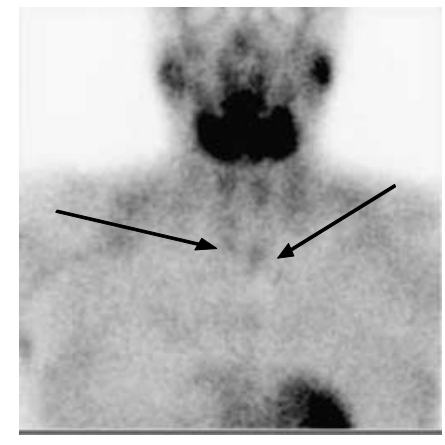

Figure 2 Clinical and genetic studies. (A) The patient has had a satisfactory growth velocity and she has reached a normal adult height with no bone deformities in contrast to her mother. (B) The L305P (c.914T >C) sequence variant was found to co-segregate with disease in the family. (C) A representative tissue section from the kidneys of the patient. The section was stained with $\mathrm{H}-\mathrm{E}$. Calcium deposits were observed in the cortical area and mainly in the interstitium. There was interstitial inflammation and fibrosis, periglomerular fibrosis but no glomerular inflammation and no vascular sclerosis. (D) The scintigraphic investigation revealed two lesions caudally of the right thyroid lobe and a lesion $1-2 \mathrm{~cm}$ caudally of the left thyroid lobe.

By 12 and a half years her calcium levels started to increase, and by 13 and a half years her phosphate levels were elevated (Table 1). By 14 years and 11 months, she was referred for hypertension and renal failure. It was noted that she recently had a reduced compliance for alfacalcidol and phosphate. She had also been diagnosed with a recent Epstein-Barr virus (EBV) infection by her family doctor. At admittance, her blood pressure was $180 \mathrm{mmHg}$ systolic to $110 \mathrm{mmHg}$ diastolic. The examination was otherwise normal. Evaluation of hypertension revealed no hypertensive retinopathy and echocardiogram revealed only a slight dilatation of the left ventricle. We identified no renovascular or endocrine causes of hypertension. She had a normal magnetic resonance imaging of the renal arteries, and normal hormone levels including catecholamines, aldosterone, cortisol, and thyroxine. She had also signs of decreased renal function (Fig. 1D) and her measured glomerular filtration rate (iohexol clearance) was reduced to $50 \mathrm{ml} / \mathrm{min}$ per $1.73 \mathrm{~m}^{2}$ (normally $80-125 \mathrm{ml} / \mathrm{min}$ per $1.73 \mathrm{~m}^{2}$ ). Nephrological evaluation revealed a normal diuresis $(1800 \mathrm{ml} / 24 \mathrm{~h}, 2600 \mathrm{ml} / 24 \mathrm{~h}$ ), normal urine sediment, an albumin to creatinine ratio of $4.9 \mathrm{mg} / \mathrm{mmol}$ (normally $<2 \mathrm{mg} / \mathrm{mmol}$ ), a slight glycosuria, and $\beta_{2}$ microglobinuria. She also presented with hypercalciuria (Table 1). Her hemoglobin concentration was normal $(13.4 \mathrm{~g} / \mathrm{dl})$ and ultrasound of her kidneys showed normal-sized kidneys with increased echogenicity, nephrocalcinosis grade I-II and no post-renal causes, including kidney stones. She had normal plasma renin assays and negative immunological assays except elevated EBV IgM and antistreptococcal DNAse B $(472 \mathrm{IU} / \mathrm{ml}, N<200)$ and antistreptolysin $(753 \mathrm{IU} / \mathrm{ml}$, $N<400$ ) levels. A renal biopsy showed calcium deposits in the cortical area mainly in the interstitium, interstitial inflammation and fibrosis, periglomerular fibrosis 
Table 1 Levels of serum calcium and phosphate and of calciuria.

\begin{tabular}{lccc}
\hline $\begin{array}{l}\text { Age } \\
\text { (years) }\end{array}$ & $\begin{array}{c}\text { Serum } \\
\text { calcium } \\
(\mathrm{mmol} / \mathrm{l})\end{array}$ & $\begin{array}{c}\text { Serum phosphate } \\
(\mathrm{mmol} /)^{\mathrm{a}}\end{array}$ & $\begin{array}{c}\text { Urine calcium to } \\
\text { creatinine ratio } \\
(\mathrm{mmol} / \mathrm{mmol})\end{array}$ \\
\hline 12.0 & 2.48 & 0.96 & 0.31 \\
12.5 & 2.62 & 0.99 & 0.38 \\
13.0 & 2.71 & 0.90 & 0.36 \\
13.5 & 2.63 & 1.05 & 0.31 \\
14.0 & 2.76 & 1.33 & 0.44 \\
14.5 & 2.87 & 1.53 & 0.34 \\
14.8 & 2.85 & $1.88^{\mathrm{b}}$ & 1.00 \\
14.9 & 2.89 & $2.36^{\mathrm{b}}$ & 0.59 \\
15.1 & 2.57 & $0.93^{\mathrm{c}}$ & 0.33 \\
15.3 & 2.48 & $0.60^{\mathrm{C}}$ & $\mathrm{NA}$ \\
15.8 & 2.39 & $0.57^{\mathrm{C}}$ & 0.21 \\
16.4 & 2.42 & $0.79^{\mathrm{C}}$ & 0.21 \\
16.7 & 2.51 & $0.58^{\mathrm{C}}$ & 0.14 \\
\end{tabular}

Normal reference values are: serum calcium $2.20-2.55 \mathrm{mM}$, serum phosphate $0.85-1.50 \mathrm{mM}$, urine calcium to creatinine ratio $<0.6 \mathrm{mmol} / \mathrm{mmol}$. NA, not available.

aSerum phosphate levels are prior to the morning phosphate dose except when otherwise indicated.

berum levels after the morning phosphate dose.

${ }^{\mathrm{C}}$ Oral phosphate discontinued.

but no glomerular inflammation and no vascular sclerosis (Fig. 2C).

It was concluded that the patient had a tubulointerstitial nephritis caused by calcium phosphate deposits, although it was realized that EBV infection can also lead to tubulointerstitial nephritis. She used no medication known to induce tubulointerstitial nephritis, had no signs of sarcoidosis and a normal ACE level. She also had a moderate hyperparathyroidism. It was decided to discontinue alfacalcidol, and then to restart alfacalcidol at a lower dose (Fig. 1A). The patient was started on $30 \mathrm{mg}$ of cinacalcet. Oral phosphate was tapered and then discontinued (Fig. 1A). She quickly became normotensive with conventional anti-hypertensive treatment with nifedipine and labetalol. With this combined treatment we reversed the increase of the calcium phosphate product and of creatinine (Fig. 1D), and the serum calcium and phosphate levels and calciuria were normalized (Table 1). The PTH levels remained moderately elevated (Fig. 1C) and a recent ultrasound of the thyroid region revealed two hypoechogenic lesions caudally of the right thyroid lobe and a lesion 1-2 cm caudally of the left thyroid lobe. Radionucleotide scanning with $99 \mathrm{mTc}$-sestamibi revealed lesions in the identical positions as demonstrated by ultrasound (Fig. 2D). At present she has reached a normal adult height of $172 \mathrm{~cm}$ and has no bone deformities in contrast to her mother (Fig. 1A).

\section{Discussion}

We have described a patient with XLH, caused by the novel missense mutation L305P in the PHEX gene. Co-segregation studies support a causal role of this variant and confirms the diagnosis of XLH. On treatment with alfacalcidol and oral phosphate the patient had normal growth and minimal bone deformities, but subsequently developed hyperparathyroidism, hypercalcemia, renal failure, and hypertension. We believe that the patient had secondary hyperparathyroidism in her first decade, which has stimulated the development of tertiary hyperparathyroidism by the age of 12 years, observed by the increase in serum calcium levels in face of elevated PTH levels. Consequently, hypercalcemia has stimulated the development of hypertension by the possible mechanism of intracellular Ca deposition in vascular smooth muscle cells (5). Normal plasma renin levels add to the evidence against a renal cause of hypertension.

Simultaneously, the patient developed renal dysfunction, as evidenced not only by the increasing serum creatinine levels and by a reduced measured glomerular filtration rate, but also by the increasing serum phosphate levels in face of a reduced compliance of oral phosphate intake. The patient had stable nephrocalcinosis, but there is no reported evidence that nephrocalcinosis leads to permanently reduced renal function $(3,10)$. Nephrocalcinosis is found in most XLH patients treated with oral phosphate and vitamin D preparation (3), and phosphate dose seems to be important for the severity of nephrocalcinosis (3). With concomitant hyperparathyroidism causing hypercalcemia and hypercalciuria, however, intratubular calcium phosphate crystals form (11), and we believe that this process has occurred in the patient as evidenced by the increase in calcium phosphate products. This process may have caused a transient renal failure, accentuated by a vicious circle where increasing renal dysfunction leads to a further rise in serum phosphate levels (10).

By reducing the intake of oral phosphate and vitamin D preparation and by adding a small dose of cinacalcet, we reversed the increase in calcium phosphate products and improved the renal function in the patient. Cinacalcet is a calcimimetic drug that modulates the calcium sensing receptor enhancing its sensitivity to circulating calcium concentrations and consequently reducing PTH secretion. It represents a new treatment of hyperparathyroidism, particularly in end stage renal failure, and is usually well tolerated (12). There is some preliminary evidence that it can reduce PTH secretion in response to a phosphate bolus in subjects with XLH (9). The patient has, however, developed myalgia, which may be related to cinacalcet-induced transient hypocalcemia (12), although most patients with cinacalcetinduced hypocalcemia will be asymptomatic (13). If unavoidable, the patient will probably require parathyroid gland surgery to correct her tertiary hyperparathyroidism.

In retrospect, we could have potentially prevented the development of tertiary hyperparathyroidism by increasing the doses of active vitamin D, allowing calciuric levels to occur which were closer to the hypercalciuria 
threshold. Alternatively, we could have reduced the absolute dose of oral phosphate or given the phosphate more frequently, even eight times daily. We would then have had to balance this approach with an increased risk of affecting normal growth and bone structure, warranting a stricter surveillance of growth and of rickets.

In conclusion, it is essential to minimize the hyperparathyroidism in XLH patients, and cinacalcet represents a new means of reducing the hyperparathyroidism and hypercalcemia. Hypercalcemia may contribute to the development of hypertension and transient renal dysfunction, and we advocate that serial measurements of blood pressure and renal function be included in the monitoring of XLH patients. Future treatment of XLH may include the direct targeting of FGF23 levels.

\section{Acknowledgements}

We thank Prof. Leif Bostad for providing a picture of the histopathological section.

\section{Disclosure}

This paper forms part of a European Journal of Endocrinology Supplement, supported by Ferring Pharmaceuticals. The auhtors disclose no potential conflicting relationship with Ferring. This article was subject to rigorous peer review before acceptance and publication

\section{References}

1 Quarles LD. Evidence for a bone-kidney axis regulating phosphate homeostasis. Journal of Clinical Investigation $2003 \mathbf{1 1 2}$ 642-646.

2 Jonsson KB, Zahradnik R, Larsson T, White KE, Sugimoto T, Imanishi Y, Yamamoto T, Hampson G, Koshiyama H, Ljunggren O, Oba K, Yang IM, Miyauchi A, Econs MJ, Lavigne J \& Juppner H. Fibroblast growth factor 23 in oncogenic osteomalacia and $\mathrm{X}$-linked hypophosphatemia. New England Journal of Medicine $20033481656-1663$.
3 Verge CF, Lam A, Simpson JM, Cowell CT, Howard NJ \& Silink M. Effects of therapy in X-linked hypophosphatemic rickets. New England Journal of Medicine 1991325 1843-1848.

4 Schmitt CP \& Mehls O. The enigma of hyperparathyroidism in hypophosphatemic rickets. Pediatric Nephrology 200419 473-477.

5 Alon US, Monzavi R, Lilien M, Rasoulpour M, Geffner ME \& Yadin O. Hypertension in hypophosphatemic rickets - role of secondary hyperparathyroidism. Pediatric Nephrology $2003 \mathbf{1 8}$ 155-158.

6 Nehgme R, Fahey JT, Smith C \& Carpenter TO. Cardiovascular abnormalities in patients with X-linked hypophosphatemia. Journal of Clinical Endocrinology and Metabolism $1997 \mathbf{8 2}$ 2450-2454.

7 Block GA, Martin KJ, de Francisco AL, Turner SA, Avram MM, Suranyi MG, Hercz G, Cunningham J, Abu-Alfa AK, Messa P, Coyne DW, Locatelli F, Cohen RM, Evenepoel P, Moe SM, Fournier A, Braun J, McCary LC, Zani VJ, Olson KA, Drueke TB \& Goodman WG. Cinacalcet for secondary hyperparathyroidism in patients receiving hemodialysis. New England Journal of Medicine $20043501516-1525$.

8 Alon US, Levy-Olomucki R, Moore WV, Stubbs J, Liu S \& Quarles LD. Calcimimetics as an adjuvant treatment for familial hypophosphatemic rickets. Clinical Journal of the American Society of Nephrology 20083 658-664.

9 Patriquin H \& Robitaille P. Renal calcium deposition in children: sonographic demonstration of the Anderson-Carr progression. American Journal of Roentgenology $19861461253-1256$.

10 Eddy MC, McAlister WH \& Whyte MP. X-linked hypophosphatemia: normal renal function despite medullary nephrocalcinosis 25 years after transient vitamin D2-induced renal azotemia. Bone 199721 515-520.

11 Alon U, Donaldson DL, Hellerstein S, Warady BA \& Harris DJ. Metabolic and histologic investigation of the nature of nephrocalcinosis in children with hypophosphatemic rickets and in the Hyp mouse. Journal of Pediatrics 1992120 899-905.

12 Dong BJ. Cinacalcet: an oral calcimimetic agent for the management of hyperparathyroidism. Clinical Therapeutics 200527 1725-1751.

13 de Francisco AL. New strategies for the treatment of hyperparathyroidism incorporating calcimimetics. Expert Opinion on Pharmacotherapy 20089 795-811.

Received 15 May 2008

Accepted 4 September 2008 